\title{
The Effect of Growth-factor Deficiencies upon Fermentation of Glucose by Yeasts
}

\author{
By R. H. HOPKINS and R. J. PENNINGTON \\ Department of Industrial Fermentation, University of Birmingham
}

\begin{abstract}
SUMMARY : The power of fermentation by yeast cells was decreased when the cultures were grown in media deficient in any of seven essential growth factors. A rapid increase in the rate of fermentation upon the addition of a growth factor was obtained in every case except that of inositol, where the response was much slower. The absence of a nitrogen source decreased the response to biotin, pantothenic acid or pyridoxin to a much greater extent than to aneurin or nicotinic acid. Differences were noted also in the behaviour of the two groups towards sodium azide, which can be explained on the assumption that biotin, pantothenic acid and pyridoxin affect fermentation only in a relatively indirect manner.

The decrease in the power of fermentation produced by pyridoxin deficiency was not observed until the pyridoxin content of the cells was only about one-fifth of the normal. Such yeast showed also an enhanced Pasteur effect. Pyridoxin deficiency produced no decrease in the proportion of glucose assimilated by resting cells.
\end{abstract}

The recognition of the importance of members of the vitamin $B$ complex in the growth of yeast is of long standing. Ample data are now available concerning the requirements of a wide range of species for individual members of the group (e.g. Burkholder, McVeigh \& Moyer, 1944; Emery, McLeod \& Robinson, 1946), and, of course, some of these requirements form the basis of microbiological assays in common use. It is now known that if one or more of the following compounds or their analogues, biotin, pantothenic acid, pyridoxin, aneurin, nicotinic acid, inositol, or $p$-aminobenzoic acid, is absent from the growth medium, certain species show a partial or complete restriction of growth.

Two of these compounds, aneurin and nicotinic acid, are involved in the generally accepted scheme of alcoholic fermentation. The exact significance of the others is less clearly understood. Few studies have been reported of the direct effect of the lack of essential growth factors upon the power of fermentation. Observations of the effect upon $\mathrm{CO}_{2}$ production in a medium otherwise adequate for growth (e.g. Atkin, Schultz \& Frey, 1946) may be difficult to interpret, since any differences may result merely from different concentrations of yeast, due to differing growth rates. Williams, Mosher \& Rohrman (1936) showed that a deficiency of pantothenic acid diminished the rate of fermentation, and a similar effect resulting from biotin deficiency has also been demonstrated and investigated in some detail (Winzler, Burk \& du Vigneaud, 1944). The results presented here concern observations made with yeasts deficient in each of the seven above-mentioned essential growth factors.

\section{METHODS}

The effects of biotin and pantothenic acid deficiencies upon fermentation were studied with a strain of Saccharomyces carlsbergensis which required these factors for growth. A strain of Kloeckera brevis (No. Y 915 of the U.S. Northern 
Regional Research Laboratory, kindly supplied by Dr L. J. Wickerham) was used for observations with aneurin, nicotinic acid and inositol. Pyridoxin deficiency was studied in both these yeasts. A strain of Saccharomyces cerevisiae requiring $p$-aminobenzoic acid (Rainbow, 1948) made possible the study of this compound.

Stock cultures were grown for $24 \mathrm{hr}$. at $25^{\circ}$ on malt extract agar enriched with $2 \%$ of glucose. At monthly intervals a transfer was made into liquid malt extract $+2 \%$ of glucose, and after growing at $25^{\circ}$ for $24 \mathrm{hr}$. a fresh agar slope culture was made from this. There was no evidence of appreciable alteration in the vigour of growth of the subcultures made from the slopes during the whole period of the work.

\section{Media and culture methods}

All measurements of fermentation were carried out using washed cells which had been grown under conditions made as uniform as possible. The complete growth medium, from which appropriate deficient media were derived by omission, or decrease to suboptimal levels, of one of the growth factors, was as follows (the amounts given are per ml.): glucose, $50 \mathrm{mg}$.; vitamin-free casein hydrolysate, $1 \mathrm{mg}$.; $\left(\mathrm{NH}_{4}\right)_{2} \mathrm{SO}_{4}, 2 \mathrm{mg}$; $\mathrm{KH}_{2} \mathrm{PO}_{4}, 1 \mathrm{mg}$.; $\mathrm{NaCl}, 0.9 \mathrm{mg}$; $\mathrm{MgSO}_{4} .7 \mathrm{H}_{2} \mathrm{O}, 0 \cdot 125 \mathrm{mg}$; $\mathrm{CaCl}_{2} .6 \mathrm{H}_{2} \mathrm{O}, 0 \cdot 185 \mathrm{mg}$; $\mathrm{MnSO}_{4} .4 \mathrm{H}_{2} \mathrm{O}, 1 \mu \mathrm{g}$.; $\mathrm{FeCl}_{3} .6 \mathrm{H}_{2} \mathrm{O}, \mathrm{ZnSO}_{4}, \mathrm{H}_{8} \mathrm{BO}_{3}, \mathrm{Tl}_{2} \mathrm{SO}_{4}, \mathrm{KI}, \mathrm{CuSO}_{4} .5 \mathrm{H}_{2} \mathrm{O}$, each $0.5 \mu \mathrm{g}$; pyridoxin, $1 \mu \mathrm{g}$.; aneurin, 0.25 $\mu \mathrm{g}$.; inositol, $25 \mu \mathrm{g}$.; D-pantothenic acid (Ca salt), 2.5 $\mu \mathrm{g}$.; nicotinic acid, 2.5 $\mu \mathrm{g}$.; DL-biotin, $0.0075 \mu \mathrm{g}$. Citric acid-potassium citrate buffer $(0.02 \mathrm{M}, \mathrm{pH} 4.5)$ was also included.

In addition, $p$-aminobenzoic acid was included in the medium for use with $S$. cerevisiae, in the concentrations given below. Pantothenic acid referred to as a medium constituent means always the $\mathrm{Ca}$ salt of $\mathrm{D}$-pantothenic acid. All the concentrations given are those realized after the addition of the yeast suspension as described below. Riboflavin (0.25 $\mu \mathrm{g} . / \mathrm{ml}$.) or pteroylglutamic acid $(0.02 \mu \mathrm{g} . / \mathrm{ml}$.), tested with $S$. carlsbergensis and $\boldsymbol{K}$. brevis, had no effect upon the growth rates.

For growing cultures for the fermentation measurements, a transfer was made from the stock culture into $10 \mathrm{ml}$. of complete medium $+1 \mathrm{ml}$. of malt extract, and grown for $24 \mathrm{hr}$. at $30^{\circ}$. The yeast cells were washed twice with sterile $0.9 \%$ saline containing also the same concentrations of other inorganic salts (except $\left.\left(\mathrm{NH}_{4}\right)_{8} \mathrm{SO}_{4}\right)$ as the growth medium; after resuspension, the concentration was adjusted turbidimetrically to exactly $20 \mu \mathrm{g}$. (dry wt.)/ml. Separate batches of $22.5 \mathrm{ml}$. of the appropriate medium, in tightly plugged $50 \mathrm{ml}$. conical flasks, were seeded with $2.5 \mathrm{ml}$. of this suspension and incubated at $30^{\circ}$, without shaking, for the time required. The cell crop was washed twice with $\mathrm{M} / 15 \mathrm{KH}_{2} \mathrm{PO}_{4}$ containing the other inorganic salts (except $\left(\mathrm{NH}_{4}\right)_{2} \mathrm{SO}_{4}$ ), and a suspension of $1 \mathrm{mg}$. (dry wt.)/ml. ( $\pm 1 \%$, measured turbidimetrically) made in a similar medium. The manometric measurements were made on measured samples from such suspensions, which were stored at just above $0^{\circ}$ and never used when more than 2 days old. 


\section{Measurement of fermentation}

Micromanometers of the constant-pressure differential type mentioned by Dixon (1943) were used. The flasks (volume about $20 \mathrm{ml}$.) were shaken through a horizontal distance of $6 \mathrm{~cm}$., 85-90 times/min., in a water bath at $30 \pm 0 \cdot 1^{\circ}$. Suitable solubility corrections were made, and all gas volumes were corrected to N.T.P. All measurements of fermentation were made under commercial nitrogen, purified by bubbling through sodium pyrogallate solution; no measurable respiration occurred under these conditions.

In each run $1 \mathrm{mg}$. (dry wt.) of yeast was used and the volume of liquid in the manometer vessel (including the yeast suspension) was $2 \mathrm{ml}$. The liquid in the vessels normally contained glucose $(3 \%),\left(\mathrm{NH}_{4}\right)_{2} \mathrm{SO}_{4}(0 \cdot 25 \%), \mathrm{KH}_{2} \mathrm{PO}_{4}(\mathrm{M} / 30)$ and the other inorganic salts in the same concentrations as used in the growth. medium. Any additions or variations are mentioned below. Inclusion of growth factors, other than the one in which the yeast was deficient, had no effect upon the rate of fermentation during the period of observation (a few hours) and consequently they were omitted. Apparently the cells contained sufficient reserves of the other essential metabolites derived from the growth medium. Additions from the side-bulb to the main compartment during the run were made in aqueous solution of volume never more than $0.2 \mathrm{ml}$. Other usual manometric techniques were used. Any change in weight of the yeast during the run was determined, if required, by turbidimetric measurement of the contents of the flask at the end.

\section{Measurement of growth}

It was desired also to record growth/time curves for the yeasts in the media used for growing cultures for the fermentation measurements. These were determined by separate experiments using $5 \mathrm{ml}$. quantities of media in flatbottomed tubes, $10 \times 1.5 \mathrm{~cm}$. internal dimensions. The depth of liquid in these tubes was approximately the same as in the flasks, and by using identical conditions of growth, rates of seeding, etc., it was found that the growth rates in the two types of vessel corresponded to within c. $10 \%$. Turbidimetric measurements were made directly on these tubes, which were selected for optical uniformity. For each medium a set of tubes was used, and the amount of growth after various times (increments usually 2 or $3 \mathrm{hr}$.) obtained by measurements upon separate tubes; successive measurements were not made upon the same tube since the shaking during the measurement stimulated growth somewhat. Each measurement was made with triplicate tubes. Turbidimetric measurements were carried out directly with a Spekker absorptiometer and expressed as dry weight of yeast by means of calibration curves for each yeast. Cells were never killed to stop growth before making measurements, since it was found that treatment with heat or antiseptics caused marked increases in the turbidity of the cultures, presumably connected with a slight shrinking and darkening observed in the microscopic appearance of the cells. 


\section{Growth-factor assays}

The content of a given growth factor in the deficient and normal yeast was determined microbiologically in a few cases. 'Pyridoxin' assays were carried out with $S$. carlsbergensis as the test organism (Hopkins \& Pennington, 1947); this assay estimates the total pyridoxin + pyridoxal + pyridoxamine. In the sequel the results of such assays are reported as 'pyridoxin'. To liberate the pyridoxin complex the samples were autoclaved at $120^{\circ}$ for $5 \mathrm{hr}$. with $0 \cdot 1 \mathrm{~N}-\mathrm{H}_{2} \mathrm{SO}_{4}$. Assays of biotin and pantothenic acid were made with the same organism, with slight modifications in the technique. Suitable concentrations for the standard curves were $0-0.1 \mathrm{~m} \mu \mathrm{g}$. of biotin and $0-20 \mathrm{~m} \mu \mathrm{g}$. of pantothenic acid/ml.

\section{RESULTS}

The influence of a deficiency of any one of the seven growth factors upon the power of fermentation of the yeast in question was readily shown. Cells harvested from suitably deficient media, with one exception, gave a lower rate of $\mathrm{CO}_{2}$ production than those of the same yeast grown in the complete medium. In preliminary studies of inositol deficiency it was observed that $18 \mathrm{hr}$. cultures of $S$. carlsbergensis in medium lacking inositol actually possessed a slightly greater power of fermentation than cultures of the same age from the complete medium, although, as expected, much less yeast was harvested from the deficient medium.

The progressive change in growth rate of $S$. carlsbergensis in various media was also measured (Fig. 1).

\section{Response to addition of groroth factor}

Typical curves showing the effect of adding the appropriate growth factor upon the rate of fermentation of deficient yeasts are shown in Figs. 2 and 3. The percentage increases in the weight of yeast during the corresponding runs (90 min.) were: $B, 10 ; P A, 5 ; P, 18$ (S. carlsbergensis), 4 ( $K$. brevis); $A, 5 ; N, 11$. Obviously the increase in the rate of $\mathrm{CO}_{2}$ production in each case is far too large to be explained merely as a direct result of the increase in weight of the yeast; the rapidity of the response would also make this unlikely.

The magnitude of such responses varied with the age of the culture and the degree of deficiency, but were remarkably constant for cultures grown for the same time in any one medium. The conditions used for the above results were chosen from experience to allow a marked response. In cases where the yeast had an absolute requirement for the growth factor in question, e.g. S. carlsbergensis for pantothenic acid or $K$. brevis for inositol, it was essential to regulate the concentration of the growth factor in the culture medium so that the yeast was still appreciably growing at the time of harvesting. Otherwise the effect of the deficiency was much less reversible; the rate of fermentation, which was decreased almost to zero, was not increased, at least within a few hours, by addition of the growth factor. Such a condition of the cultures was associated with a great irregularity and variability in the shape and size of the 


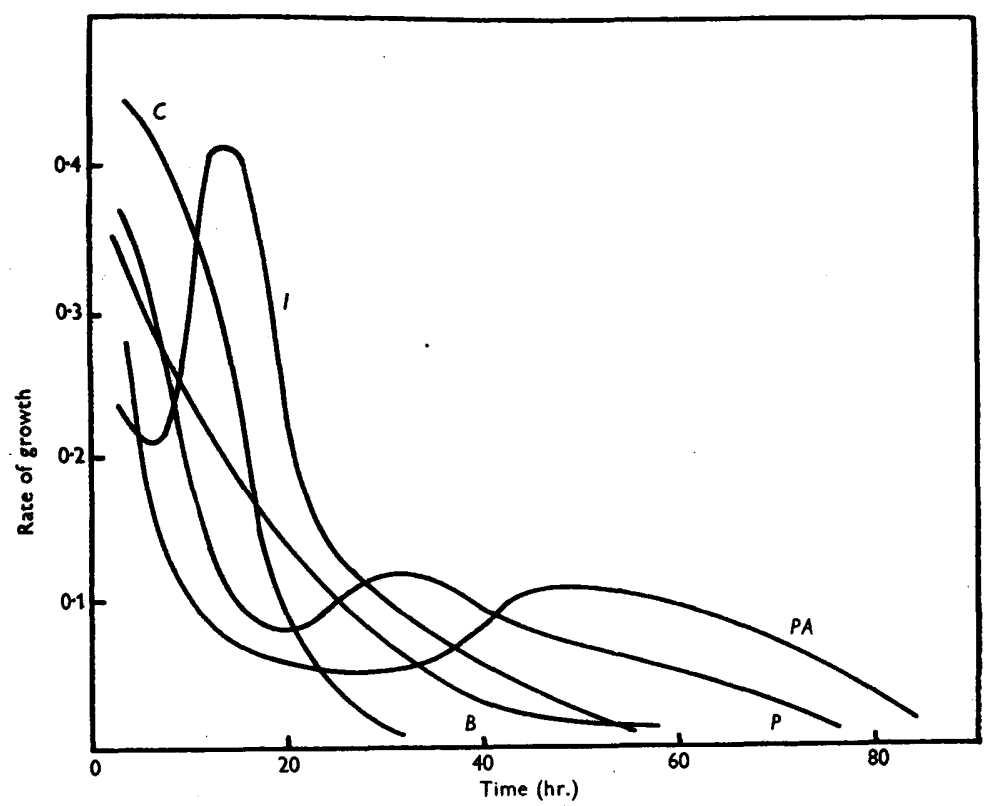

Fig. 1. Changes in growth rate of $S$. carlsbergensis with time in various media. $B$, medium without biotin; $P$, without pyridoxin; $I$, without inositol; $P A$, pantothenic acid-deficient medium; this contained $10 \mathrm{~m} \mu \mathrm{g}$. of pantothenic acid per ml., since growth was negligible in the complete absence of this factor. $C$, complete medium. Rate of growth $=\mathbf{2 \cdot 3 0 3}$ $\times\left(\log _{10} Y_{2}-\log _{10} Y_{1}\right) /\left(t_{2}-t_{1}\right)$ at time $\left(t_{1}+t_{2}\right) / 2 . \quad(Y=\mathrm{mg}$. of yeast/ml. $)$

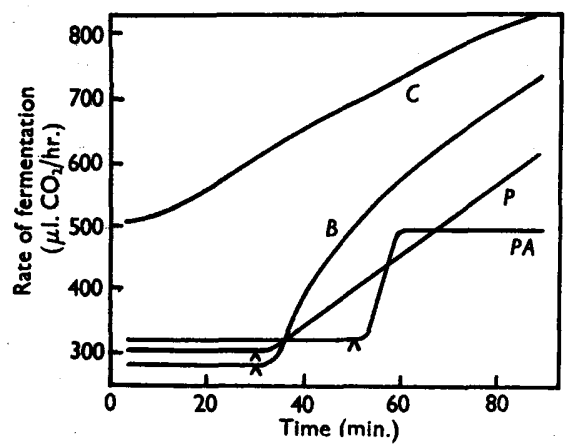

Fig. 2

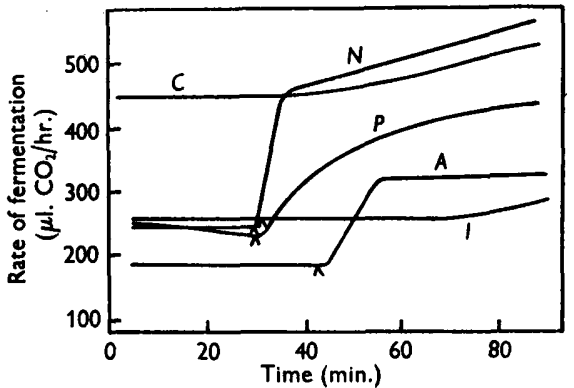

Fig. 3

Fig. 2. Response of rate of fermentation of deficient cells of $S$. carlsbergensis to addition of growth factor. $B$, biotin deficient (grown $18 \mathrm{hr}$. with no biotin); $P A$, pantothenic acid deficient (18 hr, with $40 \mathrm{~m} \mu \mathrm{g}$. pantothenic acid/ml.); $P$, pyridoxin deficient $(18 \mathrm{hr}$. with $0.5 \mathrm{~m} \mu \mathrm{g}$. pyridoxin/ml.); $C$, normal (18 hr.). The following were added from the side-bulb at the times indicated: $B, 15 \mathrm{~m} \mu \mathrm{g}$. biotin; $P A, 5 \mu \mathrm{g}$. pantothenic acid; $P, 2 \mu \mathrm{g}$. pyridoxin.

Fig. 3. Response of rate of fermentation of deficient cells of $K$. brevis to addition of growth factor. $P$, pyridoxin deficient (grown $17 \mathrm{hr}$. with $0.5 \mathrm{~m} \mu \mathrm{g}$. pyridoxin/ml.); $A$, aneurin deficient (16 hr. with $5 \mathrm{~m} \mu \mathrm{g}$. aneurin/ml.); $N$, nicotinic acid deficient (16 hr. with $20 \mathrm{~m} \mu \mathrm{g}$. nicotinic acid/ml.); I, inositol deficient $(17 \mathrm{hr}$. with $1.5 \mu \mathrm{g}$. inositol $/ \mathrm{ml}$.); $C$, normal $(17 \mathrm{hr}$.). The following were added at the times indicated: $P, 2 \mu \mathrm{g}$. pyridoxin; $A, 0.5 \mu \mathrm{g}$. aneurin; $N, 5 \mu \mathrm{g}$. nicotinic acid; $I, 50 \mu \mathrm{g}$. inositol. 
cells, with a high proportion of elongated and filamentous forms, and many dead cells, as indicated by methylene-blue staining. Biotin, pantothenic acid and 'pyridoxin' in deficient cells of $S$. carlsbergensis were assayed. These cells contained less than $10 \%$ of the amount of the respective growth factors found in the normal cells.

The lag in the response to inositol, evident in Fig. 3, was observed with cells of widely differing degrees of deficiency, and appears to be characteristic.

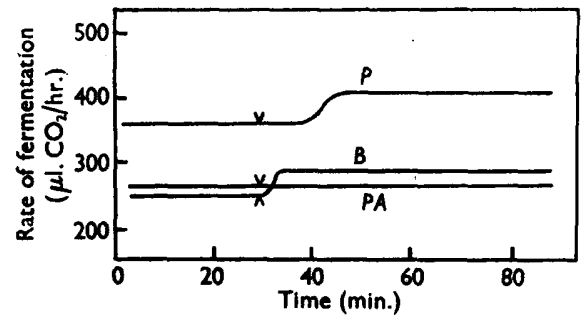

Fig. 4

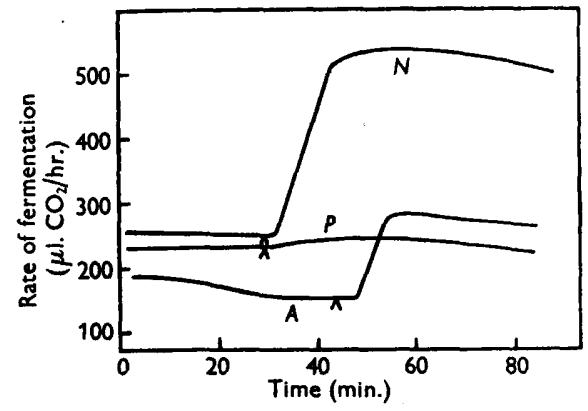

Fig. 5

Fig. 4. S. carlsbergensis. Response of deficient cells to addition of growth factor in absence of nitrogen source. For details see Fig. 2.

Fig. 5. $K$. brevis. Response of deficient cells to addition of growth factor in absence of nitrogen source. For details see Fig. 3.

The results obtained with $p$-aminobenzoic acid-deficient yeast, not included above, were complicated by experimental difficulties; the cells tended to adhere to the sides of the manometric vessels. Nevertheless, a definite, almost immediate response to the addition of the growth factor was clearly shown, as in the case of all the other deficiencies except inositol. The normal and deficient cultures of the yeast were grown for $16 \mathrm{hr}$. in the presence of 250 and $0.5 \mathrm{~m} \mu \mathrm{g} . / \mathrm{ml}$. respectively of $p$-aminobenzoic acid. (About $12 \mathrm{~m} \mu \mathrm{g} . / \mathrm{ml}$. were found to be necessary for maximum growth.)

The pantothenic acid-deficient cells of $S$. carlsbergensis responded equally rapidly to the addition of $\beta$-alanine; $40 \mu \mathrm{g}$. were added, since the potency of this substance for growth of $S$. carlsbergensis was only $1 / 20-1 / 10(w / w)$ of that of pantothenic acid. It thus appears that $\beta$-alanine can be converted immediately into pantothenic acid by this yeast.

\section{Effect of assimilable nitrogen upon the response}

In order to compare further the effect of the various growth-factor deficiencies, the results of the omission of assimilable nitrogen upon the response to each growth factor (except inositol) were studied. The experiments were carried out as before except that the medium in the manometer flasks contained no $\left(\mathrm{NH}_{4}\right)_{2} \mathrm{SO}_{4}$. Typical results are shown in Figs. 4 and 5. Results with $p$-aminobenzoic acid, not recorded, indicated a greatly decreased response. 


\section{Action of sodium azide}

In a further attempt to detect differences in the effect of the various growthfactor deficiencies upon fermentation, the action of sodium azide upon the rates was studied. Figs. 6 and 7 show the influence of sodium azide upon the rate of fermentation of yeasts deficient in various growth factors. In all cases several concentrations of azide other than that indicated were tested, but in no cases were the effects greater than that illustrated. The azide had no visible effect upon the microscopic appearance of the cells, and the proportion staining with methylene blue was much less than $1 \%$.

Figs. 8 and 9 show the effect of adding the growth factors after the addition of azide. The azide was included in the medium and the respective growth factors added at the times indicated. The final concentration of yeast was measured in all these cases; in none was there a recorded increase of more than $2 \%$, which probably does not exceed the experimental error.

The effect of azide upon the fermentation of the normal yeasts was also determined (Figs. 10 and 11). As before, several concentrations of sodium azide were tested in order to obtain the maximum stimulation. No stimulation could be observed at any concentration under the conditions of Fig. 10a; appreciably higher concentrations than that shown were inhibitory. Since yeast extract markedly increases the growth rate of $\boldsymbol{K}$. brevis on the synthetic medium (in contrast with $S$. carlsbergensis, which shows only a very slight response), another test was carried out with $K$. brevis in which $2 \mathrm{mg}$. of DIFCO yeast extract were included in the fermentation medium in addition to $\left(\mathrm{NH}_{4}\right)_{2} \mathrm{SO}_{4}$. The rate of fermentation showed a progressive rise; subsequent addition of azide (added after $40 \mathrm{~min}$. in two separate runs to produce concentrations of $10^{-4} \mathrm{M}$ and $4 \times 10^{-5} \mathrm{M}$ ) did not produce a stimulation, and in fact tended to inhibit any further rise in the rate.

\section{Other experiments with pyridoxin}

A few other aspects of pyridoxin deficiency were studied and the results are conveniently included here. $S$. carlsbergensis, grown as above, was used for all these experiments. Amongst twenty yeasts stimulated by pyridoxin, none showed relatively less growth (measured after $16 \mathrm{hr}$.) in the absence of the growth factor than this yeast.

Effect of 2:4-dinitrophenol. Some observations were made on the effect of 2:4-dinitrophenol (DNP) in comparison with azide. Typical results are shown in Fig. 12.

Variation in porver of fermentation with concentration of growth-factor. Cultures of $S$. carlsbergensis were grown $(18 \mathrm{hr}$.) with different concentrations of pyridoxin in the medium and the rate of fermentation of the washed cells measured in the usual manner. With the lower concentrations, the rate of fermentation in the manometers was constant with time; with the higher levels the rate showed a gradual increase, and in these cases the initial rate was taken. The concentration of 'pyridoxin' (= pyridoxin + pyridoxal + pyridoxamine by 
$S$. carlsbergensis assay) in the washed cells was also determined. The results are summarized in Table 1. The yield of yeast from the growth medium and the power of fermentation of the washed cells reached a maximum at c. $50 \mathrm{~m} \mu \mathrm{g}$. of

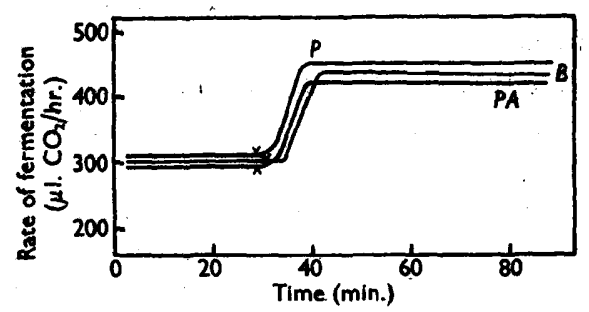

Fig. 6

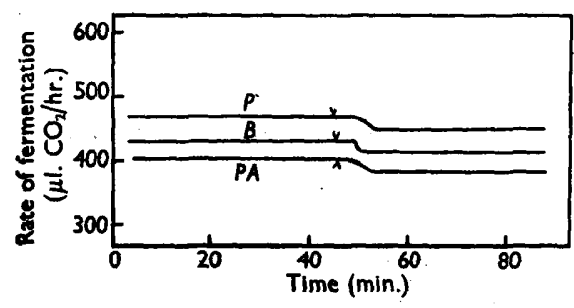

Fig. 8

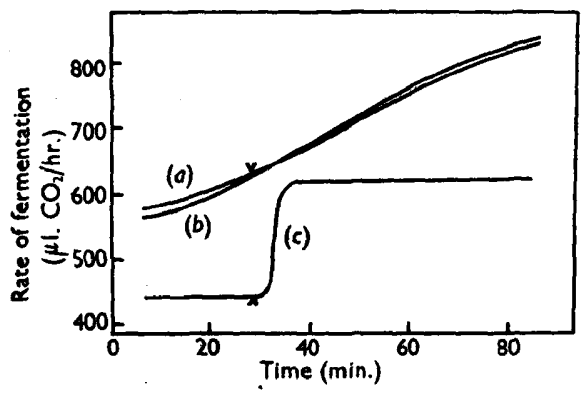

Fig. 10

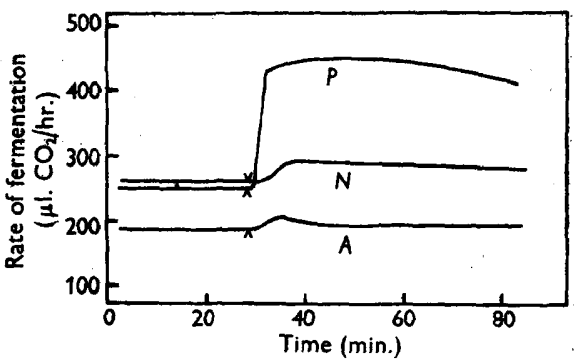

Fig. 7

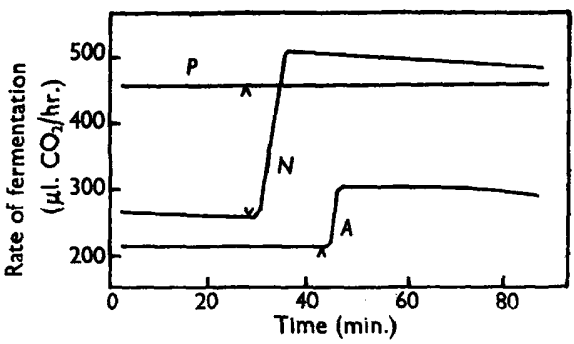

Fig. 9

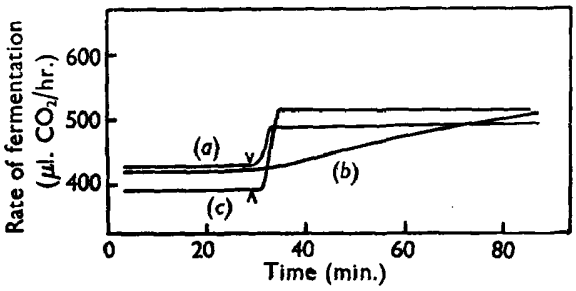

Fig. 11

Effect of sodium azide on fermentation of deficient cells.

Fig. 6. S. carlsbergensis. Azide added at points indicated to final concentration $10^{-5} \mathrm{M}$ $(B, P A) ; 10^{-4} M(P)$. Cells grown as for Fig. 2.

Fig. 7. $K$. brevis. Azide at $10^{-5} \mathrm{M}$ throughout. Cells grown as for Fig. 3 .

Fig. 8. S. carlsbergensis. Azide in medium initially at coneentrations as for Fig. 6. Growth factors added at points shown. Cells grown as for Fig. 2.

Fig. 9. $K$. brevis. Azide in medium initially at $10^{-5} \mathrm{M}$; growth factors added at points shown. Cells grown as for Fig. 3.

Fig. 10. S. carlsbergensis. Cells grown in complete medium. Azide added $(a) 2 \times 10^{-6} \mathrm{M}$; (b) no axide; (c) $2 \times 10^{-4} \mathrm{M}$. Fermentation medium (c) contained no ammonium sulphate.

Fig. 11. $\boldsymbol{K}$. brevis. Cells grown in complete medium. Azide added (a) and $(c) 4 \times 10^{-5} \mathrm{M}$; (b) no axide. Fermentation medium (c) contained no ammonium sulphate. 
pyridoxin/ml. medium, although the yeast then contained only a fraction of the amount of 'pyridoxin' present in the cells when grown with much higher concentrations of the growth factor. It was determined by cell counts that

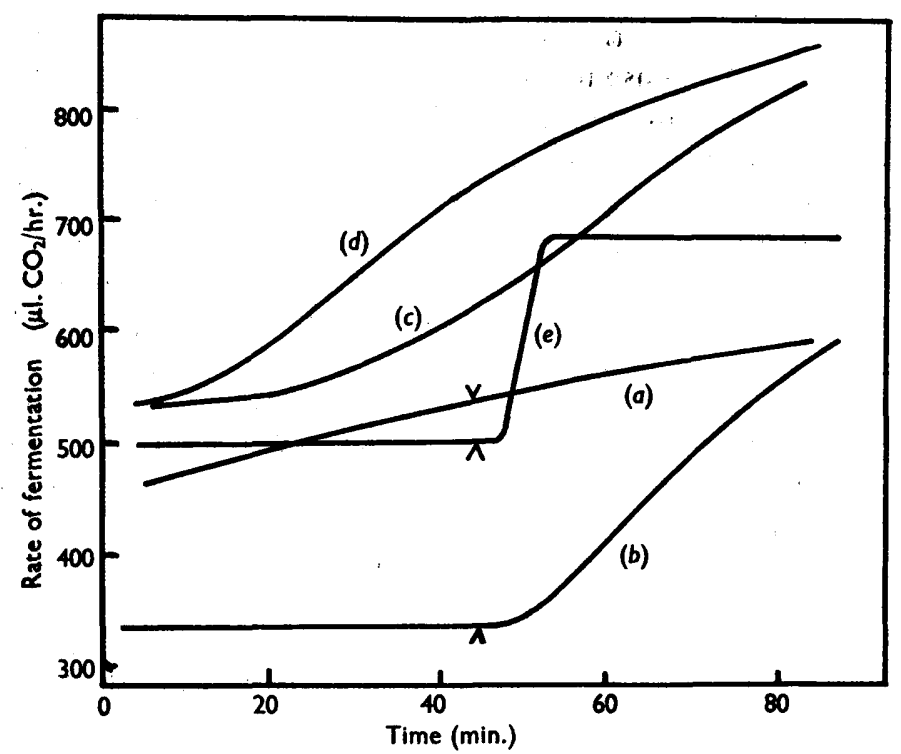

Fig. 12. Influence of 2:4-dinitrophenol (DNP) upon fermentation of pyridoxin deficient and normal cells of $S$. carlsbergensis. (a) and (b), pyridoxin deficient; $(c),(d)$ and $(e)$, normal yeast. Ammonium sulphate was omitted from the medium in $(e)$. In $(a)$ and $(c)$, DNP $\left(10^{-4} \mathrm{M}\right.$ and $5 \times 10^{-5} \mathrm{M}$ respectively) was present from the beginning. Pyridoxin was added in $(a)$ and $(b)$, and DNP (to produce a concentration of $5 \times 10^{-5} \mathrm{M}$ ) added in (e) at the points indicated.

Table 1. Yield of cells, 'pyridoxin' content, and power of fermentation of Saccharomyces carlsbergensis grown with different concentrations of pyridoxin

$\begin{array}{cccc}\begin{array}{c}\text { Pyridoxin } \\ \text { concentration } \\ \text { (m } \mu \text { g./ml.) }\end{array} & \begin{array}{c}\text { Yield of yeast } \\ \text { (mg. dry wt./ml.) }\end{array} & \begin{array}{c}\text { Pyridoxin } \\ \text { content of yeast } \\ \text { (m } \mu \mathrm{g} . / \mathrm{mg} . \\ \text { dry wt.) }\end{array} & \begin{array}{c}\text { Power of } \\ \text { fermentation } \\ \text { (percentage } \\ \text { of control) }\end{array} \\ 0 & 0 \cdot 07 & 3 \cdot 1 & 64 \\ 0 \cdot 5 & 0 \cdot 18 & 2 \cdot 6 & 66 \\ 1 \cdot 0 & 0 \cdot 36 & 2 \cdot 0 & 65 \\ 2 \cdot 0 & 0 \cdot 48 & 2 \cdot 2 & 68 \\ 3 \cdot 0 & 0 \cdot 54 & 3 \cdot 1 & 73 \\ 5 \cdot 0 & 0 \cdot 76 & 4 \cdot 5 & 77 \\ 10 \cdot 0 & 1 \cdot 02 & 5 \cdot 2 & 82 \\ 25 \cdot 0 & 1 \cdot 24 & 8 \cdot 3 & 93 \\ 50 \cdot 0 & 1 \cdot 32 & 14 \cdot 4 & 101 \\ 1000 \cdot 0 & 1 \cdot 30 & 67 \cdot 0 & 100\end{array}$

$1 \mathrm{mg}$. (dry wt.) of $S$. carlsbergensis represented c. 70 million cells. Thus the average amount of pyridoxin or its analogues in the yeast varied from just over $3 \times 10^{8}$ to c. $1 \times 10^{5}$ molecules/cell, the power of fermentation reaching its maximum at about $7.5 \times 10^{5}$ molecules/cell. 
Specificity of response to pyridoxin. In another series of experiments various substances commonly required as nutrients by micro-organisms were added from the side-bulb of the manometer flasks during fermentation by pyridoxindeficient yeast; a response not shown also by normal yeast would have suggested a metabolic relationship of the substance with pyridoxin. The following substances produced no response in the rate of fermentation: a mixture of panto-

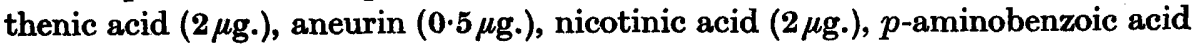
$(2 \mu \mathrm{g}$.$) , biotin (0.01 \mu \mathrm{g}$.) and inositol (25 $\mu \mathrm{g}$.); the same mixture, with the addition of pteroylglutamic acid $(0.05 \mu \mathrm{g}$ ) ; vitamin-free casein hydrolysate, adjusted to pH 4.5 (50 mg.) + DL-tryptophan (2 mg.); aspartic acid alone (2 mg.); $50 \mu \mathrm{g}$. each of adenine, guanine, uracil and xanthine; $50 \mu \mathrm{g}$. of choline chloride. Pyridoxal and pyridoxamine produced a response equal in rapidity and magnitude to that of pyridoxin: The same applied if the three analogues were added in limiting concentration ( $2 \mathrm{~m} \mu \mathrm{g}$.); the magnitude of the stimulation in this case was much less than when $2 \mu \mathrm{g}$. were added. 4-Pyridoxic acid produced no response at either concentration; this is in accordance with the negligible growth activity of this compound for $S$. carlsbergensis.

Other effects of pyridoxin deficiency. Pyridoxin-deficient yeast also showed a lowered rate of oxygen consumption in air. In this case there was a delay of over half an hour in the response to the addition of the growth factor. A similar difference in the rate of response of fermentation and respiration of biotin-deficient yeast to the addition of biotin was noted by Winzler et al. (1944). Aerobic $\mathrm{CO}_{2}$ production was influenced in a like manner to anaerobic fermentation by pyridoxin deficiency; response to the growth factor was almost immediate.

The magnitude of the Pasteur effect was also measured with normal and deficient yeast. Using the numerical expression $3\left(Q_{0}^{\mathrm{N}} \delta_{\mathrm{a}}-Q_{\mathrm{O}}^{\mathrm{O}} \mathrm{o}_{\mathrm{z}}\right) / Q_{\mathrm{o}}$, (Meyerhof oxidation quotient; M.o.Q.), values of 8.5 and 10.2 were obtained for two cultures of pyridoxin-deficient yeast, and 4.4 and 3.4 for normal yeast. Ammonium sulphate was omitted from the manometer vessels in obtaining the results used for the computation of these values. The cultures used for measurements of the rates of respiration and M.O.Q. values were grown in shallow layers of medium, shaken continuously, instead of in the usual manner; otherwise all respiration rates were very low. It was noted incidentally that the relative yield of yeast in the pyridoxin-deficient medium was less under these conditions.

The influence of pyridoxin deficiency upon the proportion of glucose assimilated during fermentation by 'resting cells' was also investigated. It is known that many micro-organisms, even in the absence of a nitrogen source necessary for growth, do not completely oxidize or ferment added substrate, but assimilate a proportion of it (Clifton, 1946); this assimilated substrate can usually be accounted for, at least in part, by an increased carbohydrate content of the cells. (Subsequently this may be broken down at a slow rate by the organism, in the absence of further substrate.) For this experiment the glucose was tipped from the side-bulb into the yeast suspension after the manometer taps were closed; in this way the total $\mathrm{CO}_{2}$ production from the glucose present was recorded. (Under anaerobic conditions, the cultures did not produce a 
measurable amount of $\mathrm{CO}_{2}$ in the absence of a substrate.) Only 1 or $2 \mathrm{mg}$. of pure, dry glucose were added; in other respects the medium was the same as usual, except that ammonium sulphate was omitted. When $1 \mathrm{mg}$. of glucose was added, the amount of $\mathrm{CO}_{2}$ produced as a percentage of the theoretical by normal and deficient yeast was 77 and 72 respectively; with $2 \mathrm{mg}$., the corresponding figures were 78 and 74. It thus appears that pyridoxin deficiency does not decrease the proportion of sugar assimilated under these conditions. The weight increases corresponding to the above figures were $0 \cdot 10,0 \cdot 15,0 \cdot 20$ and $0.26 \mathrm{mg}$. respectively. Since the increases were due presumably to carbohydrate assimilation and not to growth in the normal sense, these figures, deduced as usual from turbidimetric measurement, are probably not correct, but may have relative significance. It was also confirmed that sodium azide inhibits such assimilation; over $90 \%$ of the glucose was fermented in the presence of this substance $\left(10^{-8} \mathrm{M}\right)$.

\section{DISCUSSION}

In considering differences between the growth factors in their effects upon fermentation there is little point in comparing the magnitudes of the decreases in fermentation.rates observed; these depend upon a variety of factors, such as age of culture, or whether the yeast is completely or only partly exacting for the growth factor in question. The relative power of fermentation of cells of the same species from any of the media studied was roughly in the ratio of the growth rates at the time of harvesting, deduced from the measured growth/time curves. It may be seen from Fig. 1 that the growth rate of $S$. carlsbergensis in the complete medium showed a progressive decrease with time. The absence of an appreciable lag phase was presumably because the media were inoculated with actively growing cultures. Thorne (1939) also noted a progressive decrease in growth rate of yeast cultures. Where the medium lacked pyridoxin or inositol, however, or was low in pantothenic acid (there was no measurable growth in the complete absence of pantothenic acid), an actual increase in the growth rate occurred at one period, followed by a further decrease. This was not due to a true lag phase, since the increase was preceded in each case by a fall in the rate. It can probably be interpreted as an adaptation to the deficient environment. Such an effect was not shown by $\boldsymbol{K}$. brevis in any of the deficient media.

Owing to the pronounced increase in the growth rate in the inositol-deficient medium after about $10 \mathrm{hr}$, , the rate after about $18 \mathrm{hr}$. was actually higher than that in the complete medium; growth rate in the complete medium had fallen appreciably owing to the already extensive growth. This difference, reflected in the metabolic activities, accounts for the relatively high power of fermentation of cultures of this age from the inositol-free medium.

The age factor should always be taken into account when studying the influence of conditions of growth upon the properties of micro-organisms. The 'physiological age', which may be different-depending upon the relative growth rates-for cultures grown for the same time under different conditions, may influence the property under investigation, thus providing an additional 
variable. Apart from making observations at all stages of growth, the best procedure is probably to make comparisons between cultures with the same amount of growth.

Insufficient: is known of the action of inositol to suggest a reason for the striking lag in the response seen in Fig. 3. It would seem unlikely that it forms part of a simple coenzyme functioning in alcoholic fermentation. Differences between the action of the other growth factors are suggested by a consideration of the effects of sodium azide. Sodium azide, long recognized as a respiratory poison and known to inhibit cytochrome oxidase and other $\mathrm{Fe}$ enzymes, has more recently been shown, by numerous workers, to inhibit growth and various other endergonic physical and chemical activities of a wide variety of organisms at concentrations lower than those at which respiration is inhibited (e.g. Clifton, 1937; Spiegelman, 1946; Winzler, 1944). On the other hand, sufficiently low concentrations may actually stimulate respiration. Fermentation by yeast may. be stimulated by concentrations of azide sufficient to depress respiration, but is depressed at still higher concentrations. A clue to the nature of these effects has been provided by the observation (Spiegelman, Kamen \& Dunn, 1946; Rothstein, 1946) that azide tends to prevent the disappearance of inorganic phosphate which normally occurs during the utilization of sugar by the yeast cell. The former workers made the suggestion, supported by further evidence (Spiegelman \& Kamen, 1946), that azide interferes with the generation of high-energy phosphate bonds. The energy released in the breakdown processes. is somehow dissipated, instead of being transferred in the usual manner by phosphorylation in the adenosine diphosphate-triphosphate system. This would explain the inhibition of endergonic processes without a depression of $\mathrm{CO}_{2}$ production; the stimulation of $\mathrm{CO}_{2}$ production in some cases may be interpreted (Brockmann \& Stier, 1947) as being due to the release from the partial inhibition caused by the restriction of outlets for high-energy phosphate bonds.

It would thus appear that sodium azide may provide a useful means of dissociating the anabolic and catabolic phases of the cell's metabolism. It seemed possible that this effect could be utilized to reveal differences in the mechanism whereby the various growth factors influence the rate of fermentation. It is to be expected that in some cases this is merely an indirect manifestation of the action of the growth factor in processes involved in growth or other endergonic activities; that these may exercise control upon the rate of fermentation is highly probable in view of existing knowledge and ideas concerning the integration of the activities of the living cell, particularly the concept of the phosphate cycle (Lipmann, 1941). If the diminution in the rate of fermentation by a growth-factor deficiency depends upon such a mechanism, then azide, by liberating fermentation from the control of these processes, should tend to restore the rate. Likewise, a subsequent addition of the growth factor should be without effect upon the rate of fermentation. Examination of Figs. 6-9 suggests that this is the case with biotin, pantothenic acid and pyridoxin deficiencies. With aneurin or nicotinic acid deficiencies, on the other hand, the effect of azide in either respect is much less; this is in accordance with the known functions of these two factors in the reactions of fermentation. 
These conclusions are substantiated by the results in Fig. 10, which fit in with the postulated mechanism of the action of azide. Fermentation by $S$. carlsbergensis is stimulated by azide only in the absence of ammonium sulphate, i.e. when growth is restricted by lack of assimilable nitrogen. The results with $K$. brevis (Fig. 11) are less clear; ammonium sulphate decreases, but does not entirely eliminate, the effect of azide. However, as stated above, the stimulation by azide in the presence of ammonium sulphate was not observed when yeast extract was also present and may therefore have been due to a deficiency of unknown factors. In confirmation of this cells of $K$. brevis grown in the defined medium with added yeast extract showed no stimulation of fermentation by azide (see Fig. 11, curve $a$ ).

The conclusions reached above regarding the differences in the action of the growth factors in fermentation explain also the results in Figs. 4 and 5. If the enhancement of the rate of fermentation produced by the addition of biotin, pantothenic acid or pyridoxin is, as postulated, a result of the release from restrictions imposed by reactions involved in growth, it would be expected that no response would occur where growth processes are inhibited also by the absence of available nitrogen; the responses are, in fact, greatly decreased. Ammonium sulphate produces a rapid rise in the rate of fermentation of normal cultures of $\boldsymbol{S}$. carlsbergensis and $\boldsymbol{K}$. brevis fermenting in the absence of a nitrogen source; this is due presumably to a stimulation of endergonic processes.

Winzler et al. (1944) noted that the fermentation of cells of $S$. cerevisiae deficient in biotin failed to respond to the addition of biotin in the absence of a nitrogen source, and that sodium azide prevented this response. They inclined to the conclusion that biotin and available nitrogen were involved in the synthesis of certain enzymes involved in fermentation. Such an explanation, however, is insufficient to account for the stimulation produced by azide alone.

The action of sodium azide, in dissipating the energy produced in the cell by catabolic processes, appears to be characteristic of certain other cell poisons, in particular 2:4-dinitrophenol (see review by Clifton, 1946). Early experiments with frog muscle by Ehrenfest \& Ronzoni (1933) led to the conclusion that the controlling factor of lactic acid production in normal muscle is disturbed by DNP. The results in Fig 12 are similar in relevant points to those obtained with azide; the concentration of poison, however, was found to be even more critical.

Winzler et al. found that their biotin-deficient yeast displayed a quantitatively abnormal Pasteur effect; M.O.Q. values were several times larger than those for normal yeast (about 3-5 for the latter). The mechanisms of the Pasteur effect may be related simply to the fact that the overall rate of catabolism is limited by the prevailing concentration of inorganic phosphate or phosphate acceptors. Where the regeneration of these is slowed up by the restriction of synthetic processes, as suggested above to result from pyridoxin or biotin deficiency, it seems reasonable to expect that the Pasteur effect may be more pronounced. 


\section{REFERENCES}

Atkriv, L., Schultz, A. S. \& Frey, C. N. (1946). Enzymes and their Role in Wheat Technology (ed. J. A. Anderson), Chap. xI. New York: Interscience Publishers Inc.

Brockmann, M. C. \& Stier, T. J. B. (1947). Cellular mechanisms controlling rates of glucose consumption by yeast. J. cell. comp. Physiol. 29, 159.

Burkholder, P. R., McVeigh, J. \& MoYer, D. (1944). Studies on some growth factors of yeasts. J. Bact. 48,385 .

Cumron, C. E. (1987). On the possibility of preventing assimilation in respiring cells. Enzymologia, 4, 246.

CuIfton, C. E. (1946). Microbial assimilations. Advances in Enzymology, 6, 269.

Dixon, M. (1943). Manometric Methods, 2nd. ed., p. 7. Cambridge University Press.

Ehrenfest, E. \& Ronzont, E. (1933). Effect of dinitrophenol on oxidation of tissues. Proc. Soc. exp. Biol., N.Y., 31, 318.

Emery, W. B., McLeod, N. \& Robinson, F. A. (1946). Comparative microbiological assays of members of the vitamin $B$ complex in yeast and liver extracts. Biochem. J. 40, 426.

Hopkins, R. H. \& Pennington, R. J. (1947). The assay of the vitamin $B_{6}$ complex. Biochem. J. 41, 110.

LIPMANN, F. (1941). Metabolic generation and utilization of phosphate bond energy. Advances in Enzymology, 1, 99.

RAINBOW, C. (1948). p-Aminobenzoic acid a growth-factor for certain brewer's yeasts. Nature, Lond., 162, 572.

Rothstein, A. (1046). Phosphorylation in living yeast cells. J. cell. comp. Physiol. 28, 221.

Spregelman, S. (1946). The inhibition of enzyme formation. Fed. Proc. 5, 99.

SpIEgenman, S. \& Kamen, M. D. (1946). The site of uncoupling of phosphorylation from carbohydrate metabolism in the presence of $\mathrm{NaN}_{3}$. Fed. Proc. 5, 99.

Spiegelman, S., Kamen, M. D. \& DunN, R. (1946). Mechanism of azide inhibition of synthetic activity and its relation to phosphorylation. Fed. Proc. 5, 99.

Thorne, R. S. W. (1939). Mathematical representation of the course of yeast growth. J. Inst. Brew. 45, 472.

Williams, R. J., Mosher, W. A. \& Rohrman, E. (1936). The importance of 'pantothenic acid' in fermentation, respiration and glycogen storage. Biochem. J. 30, 2036.

WinzLer, R. J. (1944). Azide inhibition of anaerobic assimilation of glucose by yeast and its application to the determination of fermentable sugar. Science, 99, 327.

Winzler, R. J., Burk, D. \& dU Vigneaud, V. (1944). Biotin in fermentation, respiration, growth and nitrogen assimilation by yeast. Arch. Biochem. 5, 25. 